\title{
EDITORIAL
}

\section{Well-being in dementia and mild cognitive impairment}

In several ways, dementia is the final frontier for the march of Positive Psychiatry. In the recent months, International Psychogeriatrics has devoted two issues to the theme of Positive Psychiatry, reflective of the rise of this movement in the consciousness of the medical community as well as the public (see editorials: Haupt, 2019; Jeste, 2018). Yet, many people continue to see concepts such as flourishing, successful aging, meaning in life, wisdom, and wellbeing as not being applicable to (if not outright incompatible with) the lived experience of individuals with dementia. It is precisely for this reason that it is refreshing to see four articles in the current issue of International Psychogeriatrics extend the domain of Positive Psychiatry and apply these notions of well-being to among those who are most in need of it - the individuals with cognitive disorders.

A study from Belgium by Dewitte and colleagues (Dewitte et al., 2019) is a cross-sectional study of 138 older adults living in residential care with a diagnosis of Alzheimer's disease. The investigators used questionnaires administered in a structured interview format to measure meaning in life (using a subscale of Meaning in Life Questionnaire-Short Form), life satisfaction (modified version of Satisfaction with Life Scale), depressive symptoms (Geriatric Depression Scale), and general cognitive status (Mini-Mental State Examination or MMSE). The researchers found that meaning in life was positively correlated with life satisfaction and negatively with depressive symptoms. In addition, they noted a moderating influence of cognitive status, such that the relationship between meaning in life, life satisfaction, and depression was stronger in subjects with lower cognitive functioning. The moderating influence of cognitive status is an important variable because it seemed that, contrary to popular perception, meaning in life became more - and not less - important as cognitive functioning worsened. This is particularly important since the researchers also noted that depressive symptoms were more severe in subjects with more severe cognitive impairment. The study demonstrates that the perception of meaningful life is a critical psychological need for successful well-being in individuals with dementia.

Miller and colleagues (Miller et al., 2019) studied 42 dyads comprised of a hospitalized patient with dementia and a family caregiver (a majority of these caregivers were adult daughters). Well-being was assessed for both individuals with dementia and caregivers with Quality of Life-Alzheimer's Disease Scale (QOL-AD) and Center for Epidemiologic Studies-Depression Scale (CES-D). Strain in the relationship was measured along three dimensions: cognitive impairment (measured in individuals with dementia using MMSE), care-related strain (measured in caregivers) and relationship strain (measured in individuals with dementia as well as caregivers).

Relationship strain was associated with lower quality of life in the opposite member of the dyad. Worse cognition in the individual with dementia was associated with worse quality of life for the caregiver. The perception of relationship strain by the individual with dementia was related to depressive symptoms in the individual, and increased level of care-related strain was associated with caregiver's depressive symptoms. These findings highlight the fact that the quality of the dyadic relationship is inseparable from the well-being of individuals with dementia. Capturing a broader picture of well-being requires an assessment of well-being of both the individuals with dementia and their family caregivers, and the various strains in their relationships.

A systematic review and meta-ethnographic analysis from the UK (Bosco et al., 2019) explored the experience of agency in individuals with dementia. Based on a review of 20 selected studies, authors identified three third-order constructs relating to decision-making pathways and degree of autonomy: autonomous decision-making, shared decisionmaking, pseudo-autonomous decision-making. They also found that which mode of decision-making takes places depends on the severity of cognitive impairment, the urgency of the situation, the ability of the person to engage in metacognitive processes such as self-awareness, and the behavior of the caregiver. The authors discuss strategies to promote autonomous decision-making. These strategies target metacognitive regulation to understand and respond to the situation, and can include daily diaries, reminders, use of technology, simplifying the number of available choices to the most important ones, and use of advanced directives. The review and meta-ethnography did not delve into 
how these three modes of decision-making impact well-being and quality of life, and one hopes that future research efforts will bring further clarity with regards to the positive and negative consequences of these styles of autonomy, but it appears reasonable to hypothesize that pseudo-autonomous decisionmaking may have a negative effect on the well-being of the individual with dementia.

Finally, the paper by Farhang and colleagues (Farhang et al., 2019) is a systematic review of the benefits of mind-body interventions such as mindfulness, yoga, and Tai Chi for older adults with mild cognitive impairment. Nine trials met their inclusion criteria. The studies primarily looked at the impact of the interventions on cognitive function and daily functioning or physical functioning. Mind-body interventions were shown to improve cognitive function (such as executive function and memory) and had demonstrable positive effects on brain functional connectivity, rate of cognitive decline, physical fitness, sleep quality, and fall risk. Some studies also included outcomes related to mental health and psychological well-being with favorable results. Mindfulness-based stress reduction was shown to improve well-being, acceptance, and awareness of mild cognitive impairment and decrease stress reactivity. Kundalini yoga also had beneficial effects on depressive symptoms, apathy, and resilience. Tai Chi was noted to have beneficial effects on depression and anxiety in a similar vein.

Taken together, what are the implications of these studies for clinical practice? We need more interventions targeted at increasing meaning in life for individuals with dementia across the severity spectrum of cognitive impairment. Dewitte et al. point to one intervention in their discussion: meaningfocused reminiscence, an activity that is designed for individuals to reconnect with their own life story and narrative in a meaningful way, and has been shown to increase hope, life satisfaction, spiritual well-being, and cognition (Dewitte et al., 2019). We need to consider individuals with dementia in the context of their social cosmos, particularly the patient-caregiver dyadic relationship, and we must acknowledge that we cannot meaningfully talk about the well-being of the individual with dementia in isolation without talking about the well-being of the caregiver. We also need to be conscious of the agency of individuals of dementia, and to actively find strategies to encourage autonomous and shared decision-making. It is worth stressing that there is available an arsenal of mind-body interventions at our disposal that can be used to improve the wellbeing of patients with neurocognitive disorders (Eyre et al., 2017). These mind-body interventions should be incorporated into the standard of care for neurocognitive disorders.
Why is it so difficult for us to reconcile dementia and well-being? Perhaps because we are fighting against centuries of stigma, buried even into the etymological roots of our terminology. The word dementia comes from Latin "demens" or "de mente," translated as being "being out of one's mind." This notion of losing one's mind remains the stereotypical horror-story zombie-like image of dementia. We recognize "demented" to be a horrifying word although it shares the same root and meaning as dementia. To what extent does the use of terminology negatively influence the selfperception, well-being, and personhood of people with cognitive disorders? It was partly in view of the pejorative and stigmatizing connotations of the term that led to the renaming of dementia as major neurocognitive disorder in the fifth edition of the Diagnostic and Statistical Manual of Mental Disorders (Ganguli et al., 2011).

The "master narrative" of living with dementia (Somers, 1994) has long been dominated by destruction and degeneration, being condemned to a life of gradual loss, failure, and meaninglessness. It is important for us to realize that this narrative is not set in stone. These bleak images can and should be replaced by more positive visions (Harris and Keady, 2008). We must aspire for a society in which individuals with dementia can find meaning despite their illness and live with dignity and resilience. Clinicians, educators, researchers, and of course, the community will be crucial in the creation of such a world. The emergence of "dementiaville" or dementia care villages (Hogewey village in Netherlands being the pioneer) is illustrious of this development. These places make effective use of reminiscence and are specifically designed with the well-being of dementia patients in mind (Jenkins \& Smythe, 2013).

The origins of this line of thinking are not new. Kitwood, for instance, a pioneer in the field of dementia care, argued for the importance of personhood in dementia throughout his academic career. He recognized the importance of subjectivity and intersubjectivity in the lived dementia experience, and he presented evidence of subjective well-being even in those with severe cognitive impairment. $\mathrm{He}$ maintained that the key psychological task in dementia care is that of persevering the individual's personhood (Kitwood \& Bredin, 1992).

Personhood is embedded in agency, sentience, and in social relationships with others. Kitwood offered a list of 12 indicators of relative well-being in individuals with dementia, based on his observational experience (Kitwood \& Bredin, 1992): the assertion of desire or will, ability to experience and express a range of emotions, initiation of social contact, affectional warmth, social sensitivity, self-respect, acceptance of other dementia sufferers, 
humor, creativity and self-expression, showing evident pleasure, helpfulness, and relaxation. Kitwood noted that even patients with severe cognitive impairment can demonstrate such well-being, and that this well-being is partly a function of the social context, and the care provided can enhance or diminish the personhood of the individual with dementia.

Kitwood has also presented a conceptual model of the needs and well-being in dementia, which in recent years has been subjected to empirical validation. Kaufmann and Engel from Germany not only demonstrated the empirical relevance of Kitwood's model but were able to extend it further (Kaufmann \& Engel, 2016). Based on Kaufmann and Engel's extended model, subjective well-being in dementia can be conceptualized in terms of six domains, each with further sub-components. These six domains are comfort, attachment, inclusion, occupation, identity and agency. Such conceptual models are useful for the task of integrating the discoveries of the contemporaneous researchers of dementia and positive psychiatry.

How physicians make sense of well-being in dementia is another important aspect of the equation. Using semi-structured qualitative interviews, Vince et al. studied the meaning of living well with dementia from the perspective of psychiatrists (Vince et al., 2017). Their analysis revealed several different themes consistent with the ideas we have discussed above. Psychiatrists recognized that, despite the biological constraints posed by the underlying neurodegeneration, there is a possibility of living well with dementia, and that psychological and social aspects of people's lives are fundamental to this well-being. Well-being was conceptualized by psychiatrists in terms of being able to preserve as much of prior lifestyle as possible, keeping a sense of individual identity, and acceptance of the self as a person with dementia. They recognized that diagnostic disclosure by the physician, when it is done well, sets the scene for maintaining well-being in dementia, and that to do so, it is important to give positive but realistic messages. The psychiatrists, however, also pointed out the various ways in which the current systems of care do not prioritize wellbeing of individuals with dementia, resulting in a discrepancy between ideal care and actual care provided (Vince et al., 2017).

In recent years, emphasis has been placed on promoting supported decision-making instead of substituted decision making in individuals with serious mental illnesses as well as severe cognitive impairment. Supported decision making recognizes that individuals with cognitive disabilities have a sense of agency, that they can make important decisions related to their lives if they are provided the appropriate levels of support, and that they have a moral right to the availability of such supports (Jeste et al., 2018). Supported decision making when applied to dementia means that individual with dementia remains the ultimate decision maker but supporters trusted by the person (family, friends, caregivers) help the individual understand the problems and options, and the supporters help communicate the will and preferences of the individual to others. The findings on agency in dementia reported by Bosco et al. further underscore the need for further research on supported decision making in people with dementia (Bosco et al., 2019).

It is worth noting that these four papers are products of research efforts from several different countries: the USA (Miller et al., 2019), the UK (Bosco et al., 2019), Belgium (Dewitte et al., 2019), Chile and Portugal (Farhang et al., 2019). This demonstrates that well-being in dementia is a component of the global zeitgeist of dementia care. We hope that in the years to come there will be additional investigations from the other parts of the globe not represented in this issue.

This work was supported, in part, by the Sam and Rose Stein Institute for Research on Aging at the University of California San Diego.

\section{Funding}

AwAIS AFTAB ${ }^{1,2}$ AND DILIP v. JeSTE ${ }^{1,3,4}$

${ }^{1}$ Department of Psychiatry, University of California San Diego, San Diego, CA, USA

Email: djeste@ucsd.edu

${ }^{2}$ Veterans Affairs San Diego Healthcare System, San Diego, CA, USA

${ }^{3}$ Sam and Rose Stein Institute for Research on Aging, University of California San Diego, San Diego, CA, USA ${ }^{4}$ Department of Neuroscience, University of California San Diego, San Diego, CA, USA

\section{References}

Bosco, A., Schneider, J., Coleston-Shields, D. M., Jawahar, K., Higgs, P. and Orrell, M. (2019). Agency in dementia care: systematic review and meta-ethnography. International Psychogeriatrics, 31, 627-642. doi: 10.1017/ S1041610218001801.

Dewitte, L., Vandenbulcke, M. and Dezutter, J. (2019). Meaning in life matters for older adults with Alzheimer's disease in residential care: associations with life satisfaction and depressive symptoms. International Psychogeriatrics, 31, 607-615. doi: 10.1017/S1041610218002338.

Eyre, H. A., et al. (2017). A randomized controlled trial of Kundalini yoga in mild cognitive impairment. International 
Psychogeriatrics, 29(4), 557-567. doi: 10.1017/ S1041610216002155.

Farhang, M., Miranda-Castillo, C., Rubio, M. and Furtado, G. (2019). Impact of mind-body interventions in older adults with mild cognitive impairment: a systematic review. International Psychogeriatrics, 31, 643-666. doi: 10 $.1017 /$ S1041610218002302.

Ganguli, M., et al. (2011). Classification of neurocognitive disorders in DSM-5: a work in progress. The American fournal of Geriatric Psychiatry, 19, 205. doi: 10.1097/JGP .0b013e3182051ab4.

Harris, P. B. and Keady, J. (2008) Wisdom, resilience and successful aging: changing public discourses on living with dementia. Dementia, 7(1), 5-8. doi: 10.1177/ 1471301207085364.

Haupt, M. (2019) Improving images of aging. International Psychogeriatrics, 31(2), 159-161. doi: 10.1017/ S1041610219000036.

Jenkins, C. and Smythe, A. (2013). Reflections on a visit to a dementia care village. Nursing Older People, 25(6), 14-19. doi: 10.7748/nop2013.07.25.6.14.e478.

Jeste, D. V. (2018). Positive psychiatry comes of age. International Psychogeriatrics, 30, 1735-1738. doi: 10.1017/ S1041610218002211.
Jeste, D. V., Eglit, G. M. L., Palmer, B. W., Martinis, J. G., Blanck, P. and Saks, E. R. (2018). Supported decision making in serious mental illness. Psychiatry, 81, 28-40. doi: 10.1080/00332747.2017.1324697.

Kaufmann, E. G. and Engel, S. A. (2016). Dementia and well-being: a conceptual framework based on Tom Kitwood's model of needs. Dementia, 15, 774-788.

Kitwood, T. and Bredin, K. (1992). Towards a theory of dementia care: personhood and well-being. Ageing and Society, 12(3), 269-287. doi: 10.1017/S0144686X0000502X.

Miller, L. M., Kaye, J. A., Lyons, K. S., Lee, C. S., Whitlatch, C. J. and Caserta, M. S. (2019). Well-being in dementia: a cross-sectional dyadic study of the impact of multiple dimensions of strain on persons living with dementia and their family care partners. International Psychogeriatrics, 31, 617-626. doi: 10.1017/S104161021800203X.

Somers, M. R. (1994). The narrative constitution of identity: a relational and network approach. Theory and Society, 23, 605-649.

Vince, A., Clarke, C. and Wolverson, E. L. (2017). The meaning and experience of well-being in dementia for psychiatrists involved in diagnostic disclosure: a qualitative study. International Psychogeriatrics, 29(1), 93-104. doi: 10.1017/S1041610216001484. 